\title{
T CELL-MEDIATED HYPERSENSITIVITY TO LUMACAFTOR AND IVACAFTOR IN CYSTIC FIBROSIS
}

\author{
Aida Semic-Jusufagic ${ }^{1}$, Monday Ogese $^{2}$, Christopher Edwards ${ }^{1}$, Mark Wilkinson ${ }^{1}$, Claire \\ Nissenbaum ${ }^{1}$, Tim Lee ${ }^{1}$, Pirmohamed Munir ${ }^{2}$, and Dean Naisbitt ${ }^{2}$ \\ ${ }^{1}$ Leeds Teaching Hospitals NHS Trust \\ ${ }^{2}$ University of Liverpool
}

March 8, 2022

\begin{abstract}
We describe a case of Drug Reaction with Eosinophilia and Systemic Symptoms (DRESS) to multiple CFTR modulators and piperacillin in an 8 year old female with CF. To our knowledge this is the first reported case of delayed type, T-cell mediated hypersensitivity to multiple CFTR modulators described in literature. Our findings suggest possible cross-reactivity between CFTR modulators or multiple sensitisations, which should be taken into account in similar cases.
\end{abstract}

\section{T CELL-MEDIATED HYPERSENSITIVITY TO LUMACAFTOR AND IVACAFTOR IN CYSTIC FIBROSIS}

Clinical Letter

Word Count: 1097

To the Editor,

Cystic fibrosis (CF) is a serious genetic condition affecting the respiratory and digestive systems impacting life expectancy ${ }^{1}$. Patients with $\mathrm{CF}$ often require frequent high dose antibiotics associated with cutaneous hypersensitivity in $30-50 \%$ of patients ${ }^{2}$. The discovery of CFTR modulators has transformed CF treatment. These drugs tackle the underlying cause of the disease and prevent misfolding and proteasomal degradation of the CFTR protein ${ }^{3}$. Hypersensitivity to these novel drugs is a concern because it can prevent access to these transformative medications. We have recently reported a case of delayed-type $\mathrm{T}$ cell-mediated hypersensitivity to lumacaftor two weeks after a patient was treated with the combination of ivacaftor and lumacaftor ${ }^{4}$.

Herein, we describe a case of Drug Reaction with Eosinophilia and Systemic Symptoms (DRESS) to multiple CFTR modulators and piperacillin in an 8 year old female with CF. The patient was started on the daily fixed dose combination of ivacaftor/lumacaftor (Orkambi) midway through a two-week course of home intravenous (IV) antibiotics (piperacillin/tazobactam and tobramycin). She was also taking prophylactic flucloxacillin and a 4 week course of ciprofloxacin. Three days after the IV antibiotics were completed, and 9 days after the ivacaftor/lumacaftor had been commenced, the patient presented to hospital with a temperature up to $41^{\circ} \mathrm{C}$ and rapidly evolving mildly pruritic, polymorphic rash (initially maculopapular then more purpuric) starting from upper chest and spreading to face, trunk and limbs (figure 1A). She had prominent facial erythema, non-purulent conjunctivitis and complained of sore throat, malaise and vomiting. Following transfer to the CF centre, she had two further doses of piperacillin/tazobactam. Each time piperacillin/tazobactam was administered, her parents noted that patient's rash became more prominent. On the second day she developed prominent midfacial oedema. All the drugs were discontinued and she was 
started on IV meropenem and clindamycin. The blood tests revealed raised C-reactive protein $(277 \mathrm{mg} / \mathrm{L})$, lymphopenia $\left(0.45 \times 10^{9} / \mathrm{L}\right)$, leukopenia, eosinophilia $\left(0.77 \times 10^{9} / \mathrm{L}\right)$, thrombocytopenia $\left(110 \times 10^{9} / \mathrm{L}\right)$ and deranged liver function tests (ALT: $99 \mathrm{iu} / \mathrm{L}$ and albumin: $19 \mathrm{~g} / \mathrm{L}$ ). The patient also had a significant drop in phosphate and magnesium levels which required replacement. The tests conducted to exclude infectious causes (throat swab, blood cultures, Streptolysin O antibody titre, PCR for meningococcal, pneumococcal, enterovirus, paraechovirus, EBV, CMV and adenovirus, IgM to EBV and CMV) were all negative. Symptoms started resolving after 6 days with generalised desquamation of skin prominent on her face, palms and feet (figure 1B) which resolved on day 8. A year before the index reaction, she had suffered from anaphylaxis after administration of ceftazidime. At the time of assessment, patient was on prophylactic daily dose of flucloxacillin, which ruled it out as potential culprit. Further allergological work up included skin prick testing (SPT) with standardised dilutions of piperacillin/tazobactam $(4.5 \mathrm{mg} / \mathrm{ml})$, ciprofloxacin $(2 \mathrm{mg} / \mathrm{ml})$, ceftazidime $(2 \mathrm{mg} / \mathrm{ml})$, cefuroxime $(2 \mathrm{mg} / \mathrm{ml})$, ceftriaxone $(2 \mathrm{mg} / \mathrm{ml})$ and cefotaxime $(2 \mathrm{mg} / \mathrm{ml})$ and non-standardised dilution of ivacaftor/lumacaftor (crushed and diluted in $5 \mathrm{ml}$ of water for injection), all of which revealed negative results. Further intradermal testing (IDT) with standardised dilutions of ceftazidime $(1 / 10)$ and piperacillin/tazobactam (1/10) was negative on immediate reading after 20 minutes. However, the patient experienced delayed weal and flare reaction to SPT with ivacaftor/lumacaftor 24 hours later with a further increase at 72 hours (figure 1C) and positive IDT test to piperacillin/tazobactam after 24 hours (figure 1D). Patch testing with ivacaftor/lumacaftor was $1+$ positive on days 2 and 4 and negative in control subjects. The results were negative to piperacillin/tazobactam and ciprofloxacin.

We further assessed patient's in vitro drug specific T cell responses using LTT and IFN- $\gamma$ ELIspot. PBMC were isolated from patient's blood by density centrifugation using Lymphoprep (Alere Technologies AS, Oslo, Norway) from a sample provided 9 months after the index reaction. In the in vitro assays, PBMC proliferated in the presence of lumacaftor and secreted high levels of IFN- $\gamma$ (figure $1 \mathrm{E}$ and $\mathrm{G}$ ). Ivacaftor did not activate the patient's T-cells. Lower levels of PBMC proliferation and IFN- $\gamma$ secretion were also detected with piperacillin (figure $1 \mathrm{~F}$ and $\mathrm{H}$ ). The chemical structure of lumacaftor differs from piperacillin (figure $1 \mathrm{I}$ and $\mathrm{K}$ ), hence it is plausible that the observed T-cell responses is not due to cross-reactivity but is a result of a primary T-cell response to the individual drugs.

A year after the original reaction to lumacaftor, the patient was admitted for observed administration of tezacaftor/ivacaftor (Symkevi). Unfortunately, 7 hours after her first dose she started developing erythroderma spreading from chest to face and limbs, temperature up to $40^{\circ} \mathrm{C}$, headache, sore throat, malaise, abdominal pain and cough. She was treated with IV hydrocortisone, followed by daily $1 \mathrm{mg} / \mathrm{kg}$ oral prednisolone and was started on IVs meropenem. Her temperature dropped after 48 hours and the rash started resolving after 72 hours without desquamation. Laboratory investigations confirmed raised CRP (97 mg/L), eosinophilia (1.40 $\mathrm{x} 10^{9} / \mathrm{L}$ ), lymphopenia $\left(0.32 \times 10^{9} / \mathrm{L}\right)$ and deranged liver function tests (ALT $513 \mathrm{iu} / \mathrm{L}$, total bilirubin 34 $\mu \mathrm{mol} / \mathrm{L}$, albumin $26 \mathrm{~g} / \mathrm{L}$ ). Further patch testing to tezacaftor/ivacaftor and ivacaftor (Kalydeco) separately using $30 \%$ dilution in soft paraffin was positive to both at day 2 and day 4 (figure $1 \mathrm{E}$ ) suggesting reactivity to ivacaftor, showing the value of patch testing in diagnosis of CFTR induced T-cell mediated cutaneous adverse drug reactions. Assessment of T cell responses using LTT and IFN- $\gamma$ ELIspot to tezacaftor and ivacaftor failed to demonstrate significant PBMC proliferation. This has demonstrated that the patient developed hypersensitivity to lumacaftor first and subsequently to ivacaftor. The discrepancy between lumacaftor and ivacaftor in vitro T-cell responses is somewhat confusing, but may relate to the known extensive metabolism of ivacaftor. It is possible that T-cell stimulatory ivacaftor metabolites are not formed in immune cell culture. Thus, further experiments are ongoing to incorporate a CYP3A4 metabolising system into the LTT.

To our knowledge this is the first reported case of delayed type, T-cell mediated hypersensitivity to multiple CFTR modulators described in literature. Our findings suggest possible cross-reactivity between CFTR modulators or multiple sensitisations, which should be taken into account in similar cases.

Given the significant improvement in CF symptoms experienced by patients receiving the CFTR modulators, it is important to continuously monitor the safety profile of these novel drugs. Hypersensitivity to any or all of the components of CFTR modulator combinations will restrict the use of these life-transforming disease- 
modifying treatments. Although desensitisation treatment was considered, given the severity and the nature of the reaction this was not deemed suitable due to the risk of triggering potentially life threatening severe cutaneous adverse reaction.

A careful assessment of the patients' medication history, in vivotests and in vitro T-cell diagnostic assays is important for identifying the particular culprit drug(s). Accurate identification of the culprit will ensure personalised patient therapy without withholding effective treatment.

Aida Semic-Jusufagic, MD, Ph.D ${ }^{1, \#}$

Monday O Ogese, Ph.D ${ }^{2 \#}$

Christopher Edwards, $\mathrm{MD}^{3}$

Mark Wilkinson, $\mathrm{MD}^{4}$

Claire Nissenbaum, $\mathrm{MD}^{3}$

Tim Lee, MD, Ph.D ${ }^{3}$

Munir Pirmohamed, MD, Ph.D ${ }^{2}$

Dean J Naisbitt. $\mathrm{PhD}^{2}$

${ }^{1}$ Paediatric Allergy, Leeds Children's Hospital, Leeds Teaching Hospitals NHS Trust, Leeds, UK.

${ }^{2}$ MRC Centre for Drug Safety Science, Department of Pharmacology and Therapeutics, Institute of Systems, Molecular and Integrative Biology, University of Liverpool, Liverpool, UK.

${ }^{3}$ Cystic Fibrosis Centre, Leeds Children's Hospital, Leeds Teaching Hospitals NHS Trust, Leeds, UK.

${ }^{4}$ Dermatology Department, Chapel Allerton Hospital, Leeds Teaching Hospitals NHS Trust, Leeds, UK.

\# ASJ and MOO contributed equally to this work.

Correspondence: Dr Aida Semic-Jusufagic

Paediatric Allergy, E floor, Martin Wing, Leeds General Infirmary, Leeds, UK, LS1 3EX.

Telephone: +44113 392 2441; Email: aida.semic@nhs.net

Funding information: The work was conducted with funding from a BBSRC/GSK Industrial Partnership award (Grant Number BB/R008108/1). The project also received core funding from the MRC Centre for Drug Safety Science (Grant Number G0700654).

Conflict of interest statement: the authors have no conflict to declare.

Acknowledgements: The authors would like to thank the patient and her parents for their consent to publish these important findings.

Keywords: Cystic fibrosis transmembrane regulator (CFTR); cystic fibrosis (CF), lymphocyte transformation test, interferon-gamma ELIspot, Drug related eosinophilia with systemic symptoms (DRESS).

\section{Reference}

1. Boucher RC. New concepts of the pathogenesis of cystic fibrosis lung disease. The European respiratory journal. 2004; 23 (1): 146-58.

2. Parmar JS, Nasser S. Antibiotic allergy in cystic fibrosis. Thorax. 2005; 60 (6): 517-20.

3. Molinski S, Eckford PD, Pasyk S, Ahmadi S, Chin S, Bear CE. Functional Rescue of F508del-CFTR Using Small Molecule Correctors. Frontiers in pharmacology. 2012; 3 : 160. 
4. Roehmel JF, Ogese MO, Rohrbach A, Mall MA, Naisbitt DJ. Drug allergy to CFTR modulator therapy associated with lumacaftor-specific CD4(+) T lymphocytes. The Journal of allergy and clinical immunology. 2021;147 (2): 753-6.

\section{Figure legend}

Figure 1. A. Targetoid eruption suggestive of erythema multiforme (day 1). B. Toxic desquamation (day 6). C.SPT-delayed reaction to ivacaftor/lumacaftor (72 hours). D.IDT-delayed reaction to piperacillin/tazobactam (Tazocin) after 24 hours. E. Positive patch test at 48 hours to tezacaftor/ivacaftor (top) and ivacaftor (bottom) F \& G. LTT. PBMC were cultured with drugs for 7 days. T-cell proliferation assessed using thymidine incorporation and beta-counter. H\&I.Interferon- $\gamma$ secretion assessed by ELISpot. J-M. Chemical structures of drugs.

\section{Figure 1.}

\section{Hosted file}

image1.emf available at https://authorea.com/users/335459/articles/559056-t-cell-mediatedhypersensitivity-to-lumacaftor-and-ivacaftor-in-cystic-fibrosis 